\title{
Controlling a Triangular Formation of Mobile Autonomous Agents
}

\author{
M. Cao \& A. S. Morse \\ Yale University \\ $\{$ m.cao, as.morse $\}$ eyale.edu
}

\author{
C. Yu \& B. D. O. Anderson \\ Australian National University \\ and National ICT Australia Ltd. \\ $\{$ brad.yu, brian. anderson\}@anu.edu. au
}

\author{
S. Dasgupta \\ University of Iowa \\ dasgupta@engineering.uiowa.edu
}

\begin{abstract}
This paper proposes a distributed control law for maintaining a triangular formation in the plane consisting of three mobile autonomous agents. It is shown that the control law can cause any initially non-collinear, positively-oriented $\{$ resp. negatively-oriented $\}$ triangular formation to converge exponentially fast to a desired positively-oriented \{resp. negativelyoriented $\}$ triangular formation. It is also shown that there is a thin set of initially collinear formations which remain collinear and may drift off to infinity as $t \rightarrow \infty$. These findings complement and extend earlier findings cited below.
\end{abstract}

\section{INTRODUCTION}

Ever since the appearance of the work of Baillieul and Suri [1] which emphasizes the potential problem of controlling a group of mobile autonomous agents in a "directed" formation containing a cycle, interest has focused on understanding this issue in depth. A formation is directed if each agent $i$ can sense only the relative position of its "co-leaders" where by an agent $i$ 's co-leaders are meant other designated agents in the formation whose distances from agent $i$ it is the responsibility of agent $i$ to maintain. Since a directed triangular formation in the plane is the simplest formation with asymmetric co-leader relations which is both rigid and contains a cycle, it is natural to consider the problem of trying to maintain a directed triangular formation. Prompted by this, we consider the problem of maintaining a directed formation of three agents in a triangle by having each agent locally control its own position so that the distance to its co-leader \{or next agent in the triangle\} is constant. This particular problem has also recently been addressed in [2] and [3]. The latter considers intuitively devised local control laws which cause each agent to move in the direction of their respective co-leaders. The former addresses a slightly different problem and uses a control law similar to that proposed in [3]. In this paper we address the same problem using control laws of a more gradient-like nature which lend themselves to a simpler and perhaps crisper analysis than do those considered in [2] and [3]. Using straightforward arguments we prove that unique solutions to the systems of nonlinear differential equations involved exist globally.

The research of Cao and Morse was supported in part, by grants from the Army Research Office and the National Science Foundation and by a gift from the Xerox Corporation. The research of Yu and Anderson was supported by National ICT Australia, which is funded by the Australian Government's Department of Communications, Information Technology and the Arts and the Australian Research Council through the Backing Australia's Ability initiative and the ICT Centre of Excellence Program. The research of Dasgupta was supported by the National Science Foundation under grants ECS-0225530 and ECS-0622017.
We explicitly characterize a closed invariant manifold $\mathcal{N}$ on which agents are collinearly positioned and show by example that in this singular case, the three agents can move indefinitely, all ultimately converging to the same constant velocity. Our main result is to show that the control we propose will cause any initially non-collinear, "positively-oriented" \{resp. negatively-oriented \} triangular formation to converge exponentially fast to a prescribed positively-oriented \{resp. negatively-oriented $\}$ triangular formation and then come to rest. This finding extend the findings of [2] and [3] and provides a different perspective. In fact, the results of [2] are very similar to those in this paper; the essential differences between the two papers lie in the controls considered, the analytical methods used to analyze them, and the degrees of completeness of the proofs given [4]. We refer the reader to [2] and [3] for additional background and references on controlling triangular formations. See also the recent thesis by Krick [5] which deals with the more general question of formation control via rigidity.

\section{TRIANGLE FORMATION}

We consider a formation in the plane consisting of three mobile autonomous agents labelled 1,2,3 where agent 1 follows 2, 2 follows 3 and 3 follows 1 . For $i \in\{1,2,3\}$, we write $[i]$ for the label of agent $i$ 's co-leader where $[1]=2$, $[2]=3$ and $[3]=1$. We assume that the desired distance

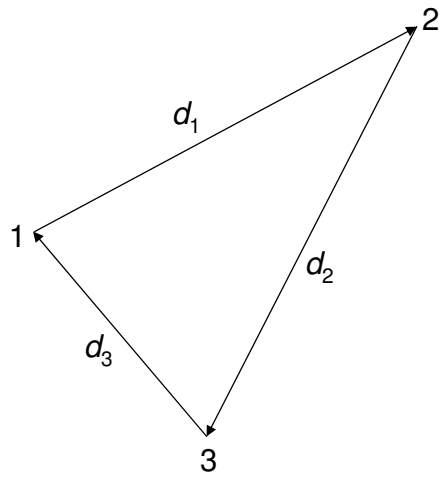

Fig. 1. Directed Point Formation

between agents $i$ and $[i]$ is $d_{i}$; here the $d_{i}$ are positive numbers which satisfy the triangle inequalities:

$$
d_{1}<d_{2}+d_{3} \quad d_{2}<d_{1}+d_{3} \quad d_{3}<d_{1}+d_{2}
$$


Note that there are two distinct triangular formations which satisfy the desired distance constraints. The first is as shown in Figure 1 and is referred to as a positively-oriented triangle. The second, called a negatively-oriented triangle, is the triangle which results when the triangle shown in Figure 1 is flipped over.

In the sequel we write $x_{i}$ for the Cartesian coordinate vector of agent $i$ in some fixed global coordinate system in the plane, and $y_{i j}$ for the position of agent $j$ in some fixed coordinate system of agent $i$ 's choosing. Thus for $i \in$ $\{1,2,3\}$, there is a rotation matrix $R_{i}$ and a translation vector $\tau_{i}$ such that $y_{i j}=R_{i} x_{j}+\tau_{i}, j \in\{1,2,3\}$. We assume that agent $i$ 's motion is described by a simple kinematic point model of the form

$$
\dot{y}_{i i}=u_{i} \quad i \in\{1,2,3\}
$$

where $u_{i}$ is agent $i$ 's control input. Thus in global coordinates,

$$
\dot{x}_{i}=R_{i}^{-1} u_{i}, \quad i \in\{1,2,3\}
$$

We assume that for $i \in\{1,2,3\}$, agent $i$ can measure the relative position of agent $[i]$ in its own coordinate system. This means that for $i \in\{1,2,3\}$, agent $i$ can measure the signal $R_{i} z_{i}$ where

$$
z_{i}=x_{i}-x_{[i]}, \quad i \in\{1,2,3\}
$$

As controls we consider

$$
u_{i}=-R_{i} z_{i} e_{i}, \quad i \in\{1,2,3\}
$$

where

$$
e_{i}=\left\|R_{i} z_{i}\right\|^{2}-d_{i}^{2}, \quad i \in\{1,2,3\}
$$

Note that the rotation matrices do not affect the definition of the $e_{i}$ in that

$$
e_{i}=\left\|z_{i}\right\|^{2}-d_{i}^{2}, \quad i \in\{1,2,3\}
$$

Moreover $R_{i}$ cancels out of the update equation

$$
\dot{x}_{i}=-z_{i} e_{i}, \quad i \in\{1,2,3\}
$$

The closed loop system of interest is thus the smooth, timeinvariant, dynamical system described in global coordinates by the equations

$$
\begin{aligned}
& \dot{x}_{1}=-\left(x_{1}-x_{2}\right)\left(\left\|x_{1}-x_{2}\right\|^{2}-d_{1}^{2}\right) \\
& \dot{x}_{2}=-\left(x_{2}-x_{3}\right)\left(\left\|x_{2}-x_{3}\right\|^{2}-d_{2}^{2}\right) \\
& \dot{x}_{3}=-\left(x_{3}-x_{1}\right)\left(\left\|x_{3}-x_{1}\right\|^{2}-d_{3}^{2}\right)
\end{aligned}
$$

In the sequel we shall refer this system as the overall system.

\section{ANALYSIS}

Our aim is to study the geometry of the overall system defined by (7)-(9). Towards this end let

$$
e=\left[\begin{array}{l}
e_{1} \\
e_{2} \\
e_{3}
\end{array}\right] \quad x=\left[\begin{array}{l}
x_{1} \\
x_{2} \\
x_{3}
\end{array}\right] \quad z=\left[\begin{array}{l}
z_{1} \\
z_{2} \\
z_{3}
\end{array}\right]
$$

First note that as a consequence of the definitions of the $z_{i}$ in (3),

$$
z_{1}+z_{2}+z_{3}=0
$$

and

$$
\begin{aligned}
& \dot{z}_{1}=-z_{1} e_{1}+z_{2} e_{2} \\
& \dot{z}_{2}=-z_{2} e_{2}+z_{3} e_{3} \\
& \dot{z}_{3}=-z_{3} e_{3}+z_{1} e_{1}
\end{aligned}
$$

Next observe that the equilibrium points of the overall system are those values of the $x_{i}$ for which

$$
z_{i} e_{i}=0, \quad i \in\{1,2,3\}
$$

Let

$\mathcal{E}$ and $\mathcal{Z}$ denote the manifolds

$$
\mathcal{E}=\{x: e=0\} \quad \mathcal{Z}=\{x: z=0\} \cup \mathcal{Q}
$$

where

$$
\mathcal{Q}=\bigcup_{i=1}^{3}\left\{x: z_{i}=0, e_{[i]}=0, e_{[[i]]}=0\right\}
$$

It is clear from (15) that every point in the manifold $\mathcal{Z} \cup \mathcal{E}$ is an equilibrium point of the overall system. The following proposition asserts that the converse is also true.

Proposition 1: The manifold $\mathcal{Z} \cup \mathcal{E}$ is the set of equilibrium points of the overall system.

Proof: Since it is clear that all points in $\mathcal{Z} \cup \mathcal{E}$ are equilibrium points of the overall system, it is enough to prove that there are no others. Towards this end, first suppose that there is an equilibrium point $\bar{x}$ at which $z_{1}=0$. It will be shown that at this point, either $z_{2}=z_{3}=0$ or $e_{2}=e_{3}=0$ and thus the only equilibrium point of the overall system at which at least one $z_{i}=0$, is a point in either $\{x: z=0\}$ or $\left\{x: z_{i}=0, e_{[i]}=0, e_{[i]]]}=0\right\}$.

The assumption that $z_{1}=0$ implies that at $\bar{x}, z_{2}=-z_{3}$ because of (11). Moreover, either $e_{2}=0$ or $z_{2}=0$ because of (15). If $z_{2}=0$, then $z_{3}=0$ because of (11); thus in this case $z_{1}=z_{2}=z_{3}=0$ and $\bar{x} \in\{x: z=0\}$. If, on the other hand, $e_{2}=0$, then either $z_{3}=0$ or $e_{3}=0$; if $z_{3}=0$ then again $z_{1}=z_{2}=z_{3}=0$. If $e_{3}=0$, then $\bar{x} \in\left\{x: z_{1}=0, e_{[1]}=0, e_{[1]]]}=0\right\}$.

To prove that the only other equilibrium points of the overall system are in $\mathcal{E}$, it is enough to show that if the $z_{i}$ are all non-zero, the only points at which (15) hold are in $\mathcal{E}$. But this is obvious. Thus we've proved that the equilibrium points of the overall system are exactly the set of points in $\mathcal{Z} \cup \mathcal{E}$.

It is easy to see that $\mathcal{Z}$ and $\mathcal{E}$ are disjoint sets. In the sequel it will be shown that $\mathcal{E}$ is attractive. It is thus not unreasonable to conjecture that all trajectories starting outside of $\mathcal{Z}$ might approach $\mathcal{E}$. However, we will give an example which illustrates that this is not the case. On the other hand, the good news is that there is another manifold containing $\mathcal{Z}$, but still not large enough to intersect $\mathcal{E}$, outside of which all trajectories approach $\mathcal{E}$. The manifold 
to which we are referring corresponds to those formations which are collinear. To explicitly characterize this manifold, we need the following fact.

Lemma 1: The points at $x_{1}, x_{2}, x_{3}$ are collinear if and only if

$$
\operatorname{rank}\left[\begin{array}{lll}
z_{1} & z_{2} & z_{3}
\end{array}\right]<2
$$

The simple proof is omitted.

To proceed, let $\mathcal{N}$ denote the set of points in $\mathbb{R}^{6}$ corresponding to agent positions in the plane which are collinear. In other words

$$
\mathcal{N}=\left\{x: \operatorname{rank}\left[\begin{array}{lll}
z_{1} & z_{2} & z_{3}
\end{array}\right]<2, z_{1}+z_{2}+z_{3}=0\right\}
$$

Note that $\mathcal{N}$ is a closed manifold containing the $\mathcal{Z}$. Although $\mathcal{N}$ contains $\mathcal{Z}$, it is still small enough to not intersect $\mathcal{E}$ :

Lemma 2: $\mathcal{N}$ and $\mathcal{E}$ are disjoint sets.

Proof: Let $x \in \mathcal{N}$ and let $z$ be correspondingly defined by (3) and (10). Since $\mathcal{Z}$ and $\mathcal{E}$ are disjoint, it is enough to show that $\mathcal{E}$ and the complement of $\mathcal{Z}$ in $\mathcal{N}$ are disjoint. Therefore suppose that $x \notin \mathcal{Z}$ in which case $z_{i} \neq 0$ for some $i \in\{1,2,3\}$. Then there must be a number $\lambda$ such that $z_{[i]}=\lambda z_{i}$. Hence $z_{[[i]]}=-(1+\lambda) z_{i}$. Suppose $x \in \mathcal{E}$; then $\left\|z_{i}\right\|=d_{i}, \quad i \in\{1,2,3\}$. Thus $|\lambda| d_{i}=d_{[i]}$ and $|1+\lambda| d_{i}=$ $d_{[[i]]}$. Then $d_{i}+d_{[i]}=d_{[[i]]}$ when $\lambda \geq 0, d_{i}+d_{[[i]]}=d_{[i]}$ when $\lambda \leq-1$, and $d_{[i]}+d_{[[i]]}=d_{i}$ when $-1<\lambda<0$. All of these equalities contradict (1). Therefore $\mathcal{N}$ and $\mathcal{E}$ are disjoint sets.

That $\mathcal{N}$ might be the place where formation control will fail is further underscored by the fact that $\mathcal{N}$ is an invariant manifold. Said differently, formations which are initially collinear, remain collinear forever. To understand why $\mathcal{N}$ is invariant, first note that for any two vectors $p, q \in \mathbb{R}^{2}$, $\operatorname{det}\left[\begin{array}{ll}p & q\end{array}\right]=p^{\prime} G q$ where

$$
G=\left[\begin{array}{cc}
0 & 1 \\
-1 & 0
\end{array}\right]
$$

From this and (11) it follows that $\operatorname{det}\left[\begin{array}{ll}z_{1} & z_{2}\end{array}\right]=$ $-\operatorname{det}\left[\begin{array}{ll}z_{1} & z_{3}\end{array}\right]$. This and the definition of $\mathcal{N}$ in (17) imply that

$$
\mathcal{N}=\left\{x: \operatorname{det}\left[\begin{array}{ll}
z_{1} & z_{2}
\end{array}\right]=0\right\}
$$

But along any solution to (12) - (14) for which (11) holds,

$$
\frac{d}{d t} \operatorname{det}\left[\begin{array}{ll}
z_{1} & z_{2}
\end{array}\right]=-\left(e_{1}+e_{2}+e_{3}\right) \operatorname{det}\left[\begin{array}{ll}
z_{1} & z_{2}
\end{array}\right]
$$

Thus if $\operatorname{det}\left[\begin{array}{ll}z_{1} & z_{2}\end{array}\right]=0$ at $t=0$, then $\operatorname{det}\left[\begin{array}{ll}z_{1} & z_{2}\end{array}\right]=0$ for all $t>0$. Therefore $\mathcal{N}$ is invariant as claimed.

It is interesting to note that $\left|\operatorname{det}\left[\begin{array}{ll}z_{1} & z_{2}\end{array}\right]\right|$ is equal to twice the area of the triangle with vertices at $x_{1}, x_{2}, x_{3}$ and for a triangle of positive area, $\operatorname{sign}\left\{\operatorname{det}\left[\begin{array}{ll}z_{2} & z_{3}\end{array}\right]\right\}$ is the triangle's orientation. A proof of these elementary claims will not be given.

Later in the paper it will be shown not only that a triangular formation cannot be achieved from an initially collinear formation, but also that there are initially collinear formations which drift off to infinity as $t \rightarrow \infty$. Despite the fact that misbehavior can occur within $\mathcal{N}$, the dimension of $\mathcal{N}$ is less than 6 which means that "almost every" initial formation will be non-collinear. The good news is that all such initial formations will converge to the desired formation and come to rest, and moreover, the convergence will occur exponentially fast. This is in essence, the geometric interpretation of our main result on triangular formations.

Theorem 1: Every trajectory of the overall system (7) (9) starting outside of $\mathcal{N}$, converges exponentially fast to a finite limit in $\mathcal{E}$.

The set of points $\mathbb{R}^{6}-\mathcal{N}$ consists of two disjoint point sets, one for which $\operatorname{det}\left[\begin{array}{ll}z_{1} & z_{2}\end{array}\right]>0$ and the other for which $\operatorname{det}\left[\begin{array}{ll}z_{1} & z_{2}\end{array}\right]<0$. Once this theorem has been proved, it is easy to verify that formations starting at points such that $\operatorname{det}\left[\begin{array}{ll}z_{1} & z_{2}\end{array}\right]<0$, converge to the positively-oriented triangular formations in $\mathcal{E}$ whereas formations starting at points such that $\operatorname{det}\left[\begin{array}{ll}z_{1} & z_{2}\end{array}\right]>0$, converge to the corresponding negatively-oriented triangular formation in $\mathcal{E}$.

The proof of Theorem 1 involves several steps. The first is to show that solutions to the overall system exist globally. To accomplish this it is especially useful to first note that the differential equations (12) - (14) together with (11) define a self-contained dynamical system since $e_{i}=\left\|z_{i}\right\|^{2}-d_{i}^{2}, \quad i \in$ $\{1,2,3\}$. This system is Lipschitz continuous and evolves on the linear manifold defined by (11). We call this system, the $Z$ - system.

Our first goal is to prove that the $z_{i}$ exist globally and are bounded. Towards this end we note that because of Lipschitz continuity, for any fixed initial value $z(0)$, there must be a largest interval $[0, T)$ on which a unique solution to (12) (14) exists. If we define

$$
V=e_{1}^{2}+e_{2}^{2}+e_{3}^{2}
$$

then along such a solution

$$
\begin{gathered}
\dot{V}=-2\left\{\left(z_{1}^{\prime} z_{1} e_{1}^{2}-z_{1}^{\prime} z_{2} e_{1} e_{2}\right)+\left(z_{2}^{\prime} z_{2} e_{2}^{2}-z_{2}^{\prime} z_{3} e_{2} e_{3}\right)\right. \\
\left.+\left(z_{3}^{\prime} z_{3} e_{3}^{2}-z_{3}^{\prime} z_{1} e_{3} e_{1}\right)\right\}
\end{gathered}
$$

or

$\dot{V}=-\left\|z_{1} e_{1}-z_{2} e_{2}\right\|^{2}-\left\|z_{2} e_{2}-z_{3} e_{3}\right\|^{2}-\left\|z_{3} e_{3}-z_{1} e_{1}\right\|^{2}$

Thus $V$ is monotone non-increasing. Since $V$ is also bounded below by $0, V$ must be bounded on $[0, T)$. In view of $V$ 's definition, the $e_{i}$ are also bounded on $[0, T)$. But boundedness of the $e_{i}$ implies boundedness of the $z_{i}$ because of (5). In particular

$$
\begin{array}{r}
\sum_{i=1}^{3}\left(\left\|z_{i}\right\|^{2}-d_{i}^{2}\right)^{2}=\sum_{i=1}^{3} e_{i}^{2} \\
\leq \sum_{i=1}^{3} e_{i}^{2}(0)=\sum_{i=1}^{3}\left(\left\|z_{i}(0)\right\|^{2}-d_{i}^{2}\right)^{2}
\end{array}
$$


This shows that each solution to the $Z$-system is bounded wherever it exists. It follows by standard arguments that $T=\infty$. We have proved the following.

Lemma 3: All trajectories of the $Z$-system exist and are bounded on $[0, \infty)$. In particular,

$$
\|z\| \leq \sqrt{2}(\|z(0)\|+\|d\|), \quad t \in[0, \infty)
$$

where $\|d\|=d_{1}+d_{2}+d_{3}$.

Notice that because of (6), Lemma 3 establishes the global existence of all solutions to the overall system.

Proposition 2: All trajectories of the overall system exist on $[0, \infty)$.

Although such solutions exist globally they need not all be bounded.

Our next goal is to show that there is an open set of points in $\mathbb{R}^{6}$ from which all solutions to the overall system tend to $\mathcal{E}$ exponentially fast. Towards this end, first observe from (20) that

$$
\dot{V}=-e^{\prime} Q^{\prime} Q e
$$

where

$$
Q=\left[\begin{array}{ccc}
-z_{1} & z_{2} & 0 \\
0 & -z_{2} & z_{3} \\
z_{1} & 0 & -z_{3}
\end{array}\right]
$$

Note that $Q$ is also the transpose of the rigidity matrix [6] of the point formation shown in Figure 1. By inspection it is clear that the rank of $Q$ is less than three just in case, for at least one distinct pair of integers $i, j \in\{1,2,3\}, z_{i}$ is a scalar multiple of $z_{j}$; moreover, because of (11) for such $i$ and $j, z_{k}$ would also have to be a scalar multiple of $z_{j}$ where $k$ is the remaining integer in $\{1,2,3\}$. In other words,

$$
\operatorname{rank} Q<3 \Longleftrightarrow \operatorname{rank}\left[\begin{array}{lll}
z_{1} & z_{2} & z_{3}
\end{array}\right]<2
$$

In the light of this and the definition of $\mathcal{N}$, it is clear that $Q^{\prime} Q$ is positive definite if and only if $x \notin \mathcal{N}$. Let $\rho$ be a positive number and define

$$
\mathcal{S}(\rho)=\left\{x: e_{1}^{2}+e_{2}^{2}+e_{3}^{3}<\rho, \quad z_{1}+z_{2}+z_{3}=0\right\}
$$

Note that $\mathcal{E} \subset \mathcal{S}(\rho)$ and that $\mathcal{S}(\rho) \longrightarrow \mathcal{E}$ as $\rho \rightarrow 0$. In view of Lemma 2 it is therefore possible to choose $\rho$ so small that $\mathcal{N}$ and the closure of $\mathcal{S}(\rho)$ are disjoint. Let $\rho$ be so chosen and let $\widehat{\mathcal{S}}$ denote the closure of $\{z: x \in \mathcal{S}(\rho)\}$. It is clear that $\widehat{\mathcal{S}}$ is compact and that $\mu\left(Q^{\prime} Q\right)>0, z \in \mathcal{\mathcal { S }}$, where for each $z \in \widehat{\mathcal{S}}, \mu\left(Q^{\prime} Q\right)$ is the smallest eigenvalue of $Q^{\prime} Q$. Thus if we define

$$
\lambda=\inf _{z \in \widehat{\mathcal{S}}} \mu\left(Q^{\prime} Q\right)
$$

then $\lambda>0$. Therefore if $x(0) \in \mathcal{S}(\rho)$, then $V(0) \leq \rho$ and $x(t) \in \mathcal{S}(\rho), t \geq 0$ because $V$ is non-increasing. This implies that $\dot{V}$ is bounded above by $-\lambda\left(e_{1}^{2}+e_{2}^{2}+e_{3}^{2}\right)$ and thus that trajectories in $\mathcal{S}(\rho)$ approach $\mathcal{E}$ as fast as $e^{-\frac{\lambda}{4} t}$.

Note that for a trajectory to converge to $\mathcal{E}$ means that the corresponding formation converges to the desired triangle. To show that the formation actually comes to rest is a simple matter of exploiting the fact that the $\left\|\dot{x}_{i}\right\|$ are bounded above by signals which are decaying to zero exponentially fast. A proof of this last observation will not be given here.

To show that all trajectories outside of $\mathcal{N}$ converge exponentially fast to $\mathcal{E}$ requires more work. In view of the preceding, we already know that any trajectory which enters $\mathcal{S}(\rho)$ in finite time must converge to $\mathcal{E}$ exponentially fast. The problem then is to show that any trajectory starting outside of $\mathcal{N}$ must enter $\mathcal{S}(\rho)$ in finite time. A key observation from (20) needed to prove this is that $\dot{V}<0$ whenever the three velocity vectors $z_{i} e_{i}, i \in\{1,2,3\}$ are not all equal. Prompted by this, let

$$
\mathcal{M}=\left\{x: x \in \mathcal{N}, z_{1} e_{1}=z_{2} e_{2}=z_{3} e_{3}\right\}
$$

Note that $\mathcal{M}$ and $\mathcal{E}$ are disjoint because $\mathcal{N}$ and $\mathcal{E}$ are.

Lemma 4: $\dot{V}=0$ if and only if $x \in \mathcal{M} \cup \mathcal{E}$.

Proof: Since it is clear that $\dot{V}=0$ whenever $x \in \mathcal{M} \cup \mathcal{E}$, we need only prove the converse. Suppose $x$ is such that $\dot{V}=0$. Then clearly, $z_{1} e_{1}=z_{2} e_{2}=z_{3} e_{3}$. It is enough to show that $x \in \mathcal{N}$ if $x \notin \mathcal{E}$. Suppose $x \notin \mathcal{E}$. Then at least one $e_{i}$ is nonzero - say $e_{1}$. Then $z_{1}$ and $z_{2}$ are linearly dependent as are $z_{1}$ and $z_{3}$. This can only occur if $x \in \mathcal{N}$.

Note that for each $x \in \mathcal{M}$, the corresponding $z$ satisfies $\dot{z}=0$, so $\mathcal{M}$ is an invariant manifold; moreover along any trajectory in $\mathcal{M}$, the three agent positions move at the same constant velocity. Thus if $\mathcal{M}$ contains any non-zero point, then the formation leaving that point will drift to infinity. The following example shows that non-zero points in $\mathcal{M}$ actually exist.

Example: Consider $d_{1}=1, d_{2}=2$ and $d_{3}=1.5$ which satisfy the triangle inequalities (1). Then one numeric solution to the equations $z_{1} e_{1}=z_{2} e_{2}=z_{3} e_{3}$ which lies in $\mathcal{M}$ is

$$
x^{*}=\left[\begin{array}{llllll}
-1.4076 & 0 & -2.0456 & 0 & 0 & 0
\end{array}\right]^{\prime}
$$

At $x^{*}, \dot{x}_{1}=\dot{x}_{2}=\dot{x}_{3}=\left[\begin{array}{lll}0.3783 & 0\end{array}\right]^{\prime}$. Using $x^{*}$ as the initial value we carry out simulations for the overall system (7) -(9). As predicted, agents 1, 2 and 3 drift to infinity in the direction of the $x$-axis.

To show that trajectories starting outside of $\mathcal{N}$ must converge exponentially fast to $\mathcal{E}$, it is enough to show that all such trajectories are bounded away from $\mathcal{M}$, even in the limit as $t \rightarrow \infty$. To understand why this is so, suppose that $x(t)$ is a trajectory starting outside of $\mathcal{N}$ and that for all $t$, $x(t)$ is bounded away from $\mathcal{M}$. Then

$$
\gamma=\inf _{t \rightarrow \infty} \delta(x(t))
$$

must be a positive number where for $x \in \mathbb{R}^{6}, \delta(x)$ denotes the distance between $x$ and $\mathcal{M}$. In view of the preceding, $x(t)$ will converge to $\mathcal{E}$ provided there is a finite time $t_{1}$ such that $x\left(t_{1}\right) \in \mathcal{S}(\rho)$. To prove that such a time must exists, we will assume the contrary and show that this leads to a contradiction.

Suppose that for all $t, x(t)$ is in the complement of $\mathcal{S}(\rho)$ which we denote by $\overline{\mathcal{S}}(\rho)$. Thus for all $t, x(t)$ is in the closed set $\mathcal{X}=\{x: \delta(x) \geq \gamma, x \in \overline{\mathcal{S}}(\rho)\}$ which in turn is 
disjoint with $\mathcal{M} \cup \mathcal{E}$. In view of Lemma $4, \dot{V}<0$ for all $t$. In addition, for all $t, z(t)$ is in the compact set $\mathcal{W}=\{z$ : $\left.x \in \mathcal{X},\|e\|^{2} \leq\|e(0)\|^{2}\right\}$; therefore $\dot{V}$ is bounded above uniformly by a negative number, namely

$$
\begin{aligned}
\sigma=-\inf _{z \in \mathcal{W}} 2\left\{\left(z_{1}^{\prime} z_{1} e_{1}^{2}-z_{1}^{\prime} z_{2} e_{1} e_{2}\right)\right. & +\left(z_{2}^{\prime} z_{2} e_{2}^{2}-z_{2}^{\prime} z_{3} e_{2} e_{3}\right) \\
+ & \left.\left(z_{3}^{\prime} z_{3} e_{3}^{2}-z_{3}^{\prime} z_{1} e_{3} e_{1}\right)\right\}
\end{aligned}
$$

Thus $V \leq V(0)-\sigma t$ for all $t$. But this is impossible because $V$ is nonnegative. Therefore $x$ enters $\mathcal{S}(\rho)$ in finite time and consequently converges to $\mathcal{E}$.

The convergence of $x$ trajectories to $\mathcal{E}$ from points outside of $\mathcal{N}$ could also be deduced from the Lasalle Invariance Principle [7] by defining the domain of definition of the original system to be $\mathbb{R}^{6}-\mathcal{N}$. However to make use of this principle one would still have to prove that trajectories starting at points outside of $\mathcal{N}$ are bounded and bounded away from $\mathcal{M}$, since the pre-compactness hypothesis of the principle demands this. Indeed, this is roughly the approach taken in [2]. An advantage of the approach taken in this paper is that it enables one to establish exponential convergence whereas, without further elaboration, the Lasalle Invarance Principle only provides asymptotic convergence.

We now turn to the problem of showing that all trajectories starting outside of $\mathcal{N}$ must be bounded away from $\mathcal{M}$, even in the limit as $t \rightarrow \infty$. As a first step toward this end, let us note that

$$
\begin{aligned}
& \operatorname{det}\left[z_{1}(t) \quad z_{2}(t)\right]= \\
& e^{-\int_{\tau}^{t}\left(e_{1}(s)+e_{2}(s)+e_{3}(s)\right) d s} \operatorname{det}\left[z_{1}(\tau) \quad z_{2}(\tau)\right] \\
& t \geq \tau \geq 0
\end{aligned}
$$

because of (19). In view of (18) it must therefore be true that any trajectory starting outside of $\mathcal{N}$ cannot enter $\mathcal{N}$ $\{$ and therefore $\mathcal{M}\}$ in finite time. It remains to be shown that any such trajectory can also not enter $\mathcal{M}$ even in the limit as $t \rightarrow \infty$. To prove that this is so we need several facts.

\section{Lemma 5:}

$$
\mathcal{N}=\mathcal{N}_{1} \cup \mathcal{N}_{2} \cup \mathcal{N}_{3}
$$

where

$\mathcal{N}_{i}=\left\{x: x \in \mathcal{N},\left\|z_{i}\right\|=\left\|z_{[i]}\right\|+\left\|z_{[i i]]}\right\|\right\}, \quad i \in\{1,2,3\}$

The simple proof of this lemma is omitted.

In view of the preceding it is possible to write

$$
\mathcal{M}=\mathcal{M}_{1} \cup \mathcal{M}_{2} \cup \mathcal{M}_{3}
$$

where $\mathcal{M}_{i}=\mathcal{N}_{i} \cap \mathcal{M}$.

Lemma 6: For any $x \in \mathcal{M}$,

$$
e_{1}+e_{2}+e_{3}<0
$$

Proof: We will prove this lemma for the case when $x \in$ $\mathcal{M}_{1}$. Following similar procedures, one can prove that the conclusion holds when $x \in \mathcal{M}_{2}$ or $x \in \mathcal{M}_{3}$. Since $x \in \mathcal{M}_{1}$, we know that $\left\|z_{1}\right\|=\left\|z_{2}\right\|+\left\|z_{3}\right\|$. Now we consider four cases:

Case 1: $\left\|z_{2}\right\|=\left\|z_{3}\right\|=0$. Then $\left\|z_{1}\right\|=\left\|z_{2}\right\|+\left\|z_{3}\right\|=0$. Thus $e_{i}=-d_{i}^{2}<0$ for $i=1,2,3$. So $e_{1}+e_{2}+e_{3}=$ $-\sum_{i=1}^{3} d_{i}^{2}<0$.

Case 2: $\left\|z_{2}\right\|=0$ and $\left\|z_{3}\right\| \neq 0$. Then $e_{2}=-d_{2}^{2}<0$, $z_{2} e_{2}=0$ and $\left\|z_{1}\right\|=\left\|z_{2}\right\|+\left\|z_{3}\right\| \neq 0$. On the other hand, in view of the definition of $\mathcal{M}$, we have $z_{1} e_{1}=z_{3} e_{3}=$ $z_{2} e_{2}=0$, so it must be true that $e_{1}=e_{3}=0$. Hence, $e_{1}+e_{2}+e_{3}=e_{2}=-d_{2}^{2}<0$.

Case 3: $\left\|z_{2}\right\| \neq 0$ and $\left\|z_{3}\right\|=0$. Similar to the discussion in case 2 , it can be shown that $e_{1}=e_{2}=0$ and $e_{3}=-d_{3}^{2}<0$. So $e_{1}+e_{2}+e_{3}=e_{3}=-d_{3}^{2}<0$.

Case 4: $\left\|z_{2}\right\| \neq 0$ and $\left\|z_{3}\right\| \neq 0$. Then $\left\|z_{1}\right\|=\left\|z_{2}\right\|+\left\|z_{3}\right\| \neq$ 0 and $\left\|z_{1}\right\|>\left\|z_{2}\right\|$. If $e_{i}=0$ for some $i \in\{1,2,3\}$, then $e_{i}=0$ for all $i \in\{1,2,3\}$ because $e_{1} z_{1}=e_{2} z_{2}=e_{3} z_{3}$ and $\left\|z_{1}\right\|,\left\|z_{2}\right\|,\left\|z_{3}\right\|>0$. However, $e_{1}, e_{2}$ and $e_{3}$ cannot be zero at the same time because agents' positions are collinear when $x \in \mathcal{M}$. Thus $e_{i} \neq 0$ for all $i \in\{1,2,3\}$. Since $e_{1} z_{1}=e_{2} z_{2}$ and $\left\|z_{1}\right\|>\left\|z_{2}\right\|$, it follows that $\left|e_{1}\right|<\left|e_{2}\right|$. Because of the definition of $\mathcal{M}_{1}$, we know that $z_{1}$ is pointing to the opposite direction with respect to that of $z_{2}$ and $z_{3}$, which implies that $e_{1} e_{2}<0$ and $e_{1} e_{3}<0$. Now suppose $e_{1}<0$. Then $e_{2}>0$ and $e_{3}>0$, which imply that $\left\|z_{1}\right\|<d_{1},\left\|z_{2}\right\|>d_{2}$ and $\left\|z_{3}\right\|>d_{3}$. Consequently $d_{1}>\left\|z_{1}\right\|=\left\|z_{2}\right\|+\left\|z_{3}\right\|>d_{2}+d_{3}$ which contradicts the triangle inequality $d_{1}<d_{2}+d_{3}$. Hence, it must be true that $e_{1}>0, e_{2}<0$ and $e_{3}<0$. In view of the fact $\left|e_{1}\right|<\left|e_{2}\right|$, we know $e_{1}+e_{2}+e_{3}<e_{3}<0$.

Considering the discussion of all these four cases, we conclude $e_{1}+e_{2}+e_{3}<0$.

We are now ready to show that any trajectory starting outside of $\mathcal{N}$, cannot approach $\mathcal{M}$ in the limit as $t \rightarrow \infty$. Suppose the opposite is true, namely that $x(t)$ is a trajectory starting outside of $\mathcal{N}$ which approaches $\mathcal{M}$ as $t \rightarrow \infty$. Then in view of (21), (18), and the fact that $\mathcal{M} \subset \mathcal{N}$,

$$
\lim _{t \rightarrow \infty}\left|\operatorname{det}\left[\begin{array}{ll}
z_{1} & z_{2}
\end{array}\right]\right|=0
$$

We will now show that this is false.

In view of Lemma 6 , there must be an open set $\mathcal{V}$ containing $\mathcal{M}$ on which the inequality in the lemma continues to hold. In view of Lemma 2 and the fact that $\mathcal{M} \subset \mathcal{N}$, it is possible to choose $\mathcal{V}$ small enough so that in addition to the preceding, $\mathcal{V}$ and $\mathcal{E}$ are disjoint. For $x(t)$ to approach $\mathcal{M}$ means that for some finite time $T, x(t) \in \mathcal{V}, t \in[T, \infty)$. This implies that $e_{1}+e_{2}+e_{3}<0, t \geq T$. In view of (21),

$$
\begin{aligned}
& \left|\operatorname{det}\left[\begin{array}{ll}
z_{1} & z_{2}
\end{array}\right]\right| \geq\left|\operatorname{det}\left[z_{1}(T) \quad z_{2}(T)\right]\right|, t \geq T \text {. But } \\
& \left|\operatorname{det}\left[z_{1}(T) \quad z_{2}(T)\right]\right|= \\
& e^{-\int_{0}^{T}\left(e_{1}(s)+e_{2}(s)+e_{3}(s)\right) d s}\left|\operatorname{det}\left[z_{1}(0) \quad z_{2}(0)\right]\right|
\end{aligned}
$$

Moreover, $\left|\operatorname{det}\left[z_{1}(0) \quad z_{2}(0)\right]\right|>0$ because $z$ starts outside of $\mathcal{N}$. Therefore $\left|\operatorname{det}\left[\begin{array}{ll}z_{1} & z_{2}\end{array}\right]\right|>\left|\operatorname{det}\left[z_{1}(T) \quad z_{2}(T)\right]\right|>$ $0, t \geq T$ which contradicts (22). This completes the proof of Theorem 1. 
The preceding proves among other things that trajectories starting outside of $\mathcal{N}$ cannot approach $\mathcal{M}$. But $\mathcal{N}$ is an invariant manifold. Moreover, we've already proved that all trajectories starting outside of $\mathcal{N}$ converge to $\mathcal{E}$. We can therefore conclude that any trajectory starting outside of $\mathcal{N}$ can never enter $\mathcal{N}$. On the other hand, any trajectory starting inside of $\mathcal{N}$ must approach $\mathcal{M}$. This can easily be proved by exploiting the fact that $\dot{V}<0$ at all points in $\mathcal{N}$ which are not in $\mathcal{M}$.

\section{CONCLUDING REMARKS}

It is likely that the findings of this paper can be shown to hold for any given rigid formation in the plane consisting of any number of autonomous agents, provided each agent admits a kinematic point model as assumed in this paper, and the distance constraints which each agent must satisfy are consistent [8]. This is suggested not only by the results derived here but also by the findings of [9] which address the distance constrained formation maintenance problem assuming small errors in agent positions. To deal with more realistic agent models, one might consider the use of virtual shells [10].

\section{REFERENCES}

[1] J. Baillieul and A. Suri. Information patterns and hedging Brockett's theorem in controlling vehicle formations. In Proc. of the 42th IEEE Conference on Decision and Control, pages 194 - 203, 2003.

[2] S. L. Smith, M. E. Broucke, and B. A. Francis. Stabilizing a multiagent system to an equilibrium polygon formation. In Proc. of the 17th MTNS, pages 2415-2424, 2006.

[3] B. D. O. Anderson, C. Yu, S. Dasgupta, and A. S. Morse. Control of a three-coleader formation in the plane. Systems and Control Letters, 56:573-578, 2007.

[4] M. Cao, C. Yu, A. S. Morse, B. D. O. Anderson, and S. Dasgupta. Generalized controller for directed triangle formations. In Proc. of the 2008 IFAC Congress, 2008. submitted.

[5] L. Krick. Application of graph rigidity in formation control of multi-robot networks. Master's Thesis, Department of Electrical and Computer Engineering, University of Toronto, 2007.

[6] T. Eren, P. N. Belhumeur, B. D. O. Anderson, and A. S. Morse. A framework for maintaining formations based on rigidity. In Proc. of the 2002 IFAC Congress, pages 2752-2757, 2002.

[7] J. P. Lasalle. The Stability of Dynamical Systems. Society for Industrial and Applied Mathematics, 1976.

[8] J. M. Hendrickx, B. D. O. Anderson, J.-C. Delvenne, and V. D. Blondel. Directed graphs for the analysis of rigidity and persistence in autonomous agent systems. Int. J. Robust Nonlinear Control, 17:960981, 2007.

[9] B. D. O. Anderson, S. Dasgupta, and C. Yu. Control of directed formations with a Leader-First Follower structure. In Proc. of the 46th IEEE Conference on Decision and Control, 2007. To be presented.

[10] A. S. Morse. Virtual shells for avoiding collisions. Presentation available on line at the URL http://entity.eng.yale.edu/controls/publications.html, jul 2001. 